Héctor H. Cuenya, Departamento de Matemática, Fac. de Cs. Exactas F. Q. y Naturales, Universidad Nacional de Río Cuarto, Campus Universitario, 5800 Río Cuarto, Argentina. e-mail: hcuenya@unrccc.edu.ar

Felipe Zó, Instituto de Matemática Aplicada San Luis, Universidad Nacional de San Luis, Conicet, 5700 San Luis, Argentina. e-mail: fzo@unsl.edu.ar

\title{
MONOTONE NORMS ON $C(\Omega)$ AND MULTIPLICATIVE FACTORS
}

\begin{abstract}
Let $C(\Omega)$ be the algebra of continuous complex-valued functions on a topological space $\Omega$ and let $\rho$ be a function norm on $C(\Omega)$. We give necessary and sufficient conditions on the set $A_{\rho}=\{f \in C(\Omega): \rho(f)<\infty\}$ to be an algebra. Also, we prove that every complete function norm is quasi-submultiplicative provided $A_{\rho}$ is an algebra and we give a characterization of the best multiplicative factor of $\rho$. Finally we characterize the infinity norm and we prove that every quasi-submultiplicative function norm on $C(\Omega)$ is equivalent to the infinity norm.
\end{abstract}

\section{Introduction}

Let $\Omega$ be a topological space and let $C(\Omega)$ be the algebra of continuous complex-valued functions. In a similar way as it was introduced in [3] we are going to consider a function norm $\rho$ on $C(\Omega)$, i.e., a function $\rho: C(\Omega) \rightarrow[0, \infty]$ which satisfies the usual properties of a norm, including the monotonicity condition

$$
f, g \in C(\Omega),|f| \leq|g| \Rightarrow \rho(f) \leq \rho(g) .
$$

It follows immediately from the definition that $\rho(|f|)=\rho(f)$ for all $f \in C(\Omega)$.

Key Words: monotone norms, submultiplicative norms, multiplicative factors, algebra of continuous functions.

Mathematical Reviews subject classification: Primary 46H05,46J10

Received by the editors June 19, 1997

* This work, was supported by CONICET, CONICOR, Univ. Nac. de Río Cuarto and Univ. Nac. de San Luis. 
Throughout this paper $A_{\rho}$ will denote the following subspace of $C(\Omega)$,

$$
A_{\rho}=\{f \in C(\Omega): \rho(f)<\infty\},
$$

and $\mathcal{B}(\Omega)$ will denote the space of bounded complex-valued functions on $\Omega$. We are interested in giving necessary and sufficient conditions on $C(\Omega)$ for existence of a function norm such that $A_{\rho}$ is an algebra. This problem was studied for other spaces in [1], and later in [2] and it is related to the existence of submultiplicative norms, see [4], [5] and [6].

A norm $\rho: C(\Omega) \rightarrow[0, \infty]$ will be called $\sigma$-subadditive if for all sequences of functions $f_{n} \in C(\Omega), f_{n} \geq 0$ and $\sum_{1}^{\infty} f_{n} \in C(\Omega)$ it follows that

$$
\rho\left(\sum_{1}^{\infty} f_{n}\right) \leq \sum_{1}^{\infty} \rho\left(f_{n}\right) .
$$

We will say that a norm $\rho$ on $C(\Omega)$ is complete if $\left(A_{\rho}, \rho\right)$ is complete. It is not difficult to see that every complete function norm on $C(\Omega)$ is $\sigma$-subadditive. See for example [3]. We will say that a norm $\rho$ is quasi-submultiplicative if there exists a constant $K>0$ such that

$$
\rho(f g) \leq K \rho(f) \rho(g),
$$

for all $f, g \in C(\Omega)$. In this case we will say that $K$ is a multiplicative factor of $\rho$. The infimum of all multiplicative factors of $\rho$ it is called the best multiplicative factor of $\rho$. Obviously given a quasi-submultiplicative function norm $\rho$, with $A_{\rho} \neq\{0\}$, its best multiplicative factor $M$ is again a multiplicative factor of $\rho$, in particular $M>0$.

One of our main results states that if $\rho$ is a $\sigma$-subadditive function norm on $C(\Omega)$ and $A_{\rho}$ is an algebra, $A_{\rho}$ can not contains an unbounded function. The following example shows that there exist $\sigma$-subadditive function norms on $C(\Omega)$ where the subspace $A_{\rho}$ admits unbounded functions. Let $\Omega$ be the interval $(0,1)$ and

$$
\rho(f)=\left(\int_{\Omega} f^{2} d x\right)^{1 / 2}=\|f\|_{2},
$$

where $d x$ stands for Lebesgue measure. Clearly, $\rho$ is a $\sigma$-subadditive function norm on $C((0,1))$. However there exist unbounded square integrable continuous functions on $(0,1)$.

We will show that a function norm $\rho$ is quasi-submultiplicative provided it is a complete norm function and $A_{\rho}$ is an algebra. For the function norm $\rho$ we are interested in obtaining an alternative, expression for the best multiplicative factor of $\rho$ which is easier to handle. Along the way we will give a 
characterization of the best multiplicative factor analogous to the one in [2] in the case of function norms defined on measurable spaces. More explicitly, we prove for any non trivial $\left(A_{\rho} \neq\{0\}\right)$ quasi-submultiplicative norm function $\rho$, that its best multiplicative factor is given by

$$
M_{\rho}=\sup \left\{\|f\|_{\infty}: f \in C(\Omega), \rho(f) \leq 1\right\},
$$

where $\|\cdot\|_{\infty}$ denote the infinity norm. Note that $M_{\rho}$ is a well defined number in $[0, \infty]$ for any function norm $\rho$ and because $M_{\rho}$ is a finite number it will characterize quasi-submultiplicative norms.

Finally, we will give a simple characterization of the infinity norm and we will prove that for every finite complete function norm $\rho$ on $C(\Omega)$ the multiples $\lambda \rho$ are submultiplicative norms for $\lambda \geq M_{\rho}$. In general it is easier to decide that a norm is monotone and complete. Then the previous result gives us a method to obtain submultiplicative norms.

\section{Results and Proofs}

Theorem 1. Let $\rho$ be a function norm on $C(\Omega)$.

(a) If $\rho$ is $\sigma$-subadditive, then $A_{\rho}$ is an algebra if and only if $A_{\rho} \subset \mathcal{B}(\Omega)$,

(b) If $A_{\rho}=C(\Omega)$, then $C(\Omega) \subset \mathcal{B}(\Omega)$.

(c) If $\rho$ is quasi-submultiplicative, then $A_{\rho} \subset \mathcal{B}(\Omega)$.

Proof. (a) We assume that $A_{\rho}$ is an algebra. Suppose that there exists an unbounded function $f \in A_{\rho}$. Since $\rho(|f|)=\rho(f)$ we assume that $f \geq 0$. The next argument is similar to the one used in Theorem 1 of [4]. Thus we get a sequence of elements $t_{n} \in \Omega$ such that $f\left(t_{n+1}\right)>f\left(t_{n}\right)+3$ for each $n \in \mathbb{N}$, $f\left(t_{1}\right)>2$ and $\frac{f\left(t_{n}\right)}{n^{2}}$ tends to infinity. Let $\left(I_{n}\right)$ be the sequence of pairwise disjoint closed intervals $I_{n}=\left[f\left(t_{n}\right)-1, f\left(t_{n}\right)+1\right]$ and for each $n \in \mathbb{N}$ we choose a continuous function $g_{n}: \mathbb{R} \rightarrow \mathbb{R}$ with $\operatorname{supp}\left(g_{n}\right) \subset I_{n} g_{n} \geq 0$ and $\left\|g_{n}\right\|_{\infty}=1$. Let $h_{n}(t)=g_{n}(f(t))$. Then $h_{n} \in C(\Omega), 0 \leq h_{n}(t) \leq f(t)$ for all $t \in \Omega$, so $h_{n} \in A_{\rho}$ and $\left(f h_{n}\right)(t) \geq\left(f\left(t_{n}\right)-1\right) h_{n}(t)$ for all $t \in \Omega$. Thus $0<\rho\left(h_{n}\right)<\infty$. Let $g=\sum_{1}^{\infty} \frac{h_{n}}{n^{2} \rho\left(h_{n}\right)}$. Clearly $g \in C(\Omega)$. Since $\rho$ is $\sigma$-subadditive, we have that $g \in A_{\rho}$. Then $f g \in A_{\rho}$. On the other hand, by the monotonicity we get

$$
\rho(f g) \geq \frac{\rho\left(f h_{n}\right)}{n^{2} \rho\left(h_{n}\right)} \geq \frac{f\left(t_{n}\right)-1}{n^{2}},
$$

which is a contradiction. 
Assume now $A_{\rho} \subset \mathcal{B}(\Omega), f$ and $g$ belong to $A_{\rho}$. Thus we have $\rho(f g) \leq$ $\|f\|_{\infty} \rho(g)<\infty$. Therefore $f g \in A_{\rho}$.

(b) Suppose that there exists an unbounded function $f \in C(\Omega)$. By defining a function $g$ as in (a), we obtain as before a contradiction.

(c) Suppose that there exists an unbounded function $f \in A_{\rho}$. We choose $g_{n}$ and $h_{n}$ as in (a). Then for some $K>0$ we get $f\left(t_{n}-1\right) \rho\left(h_{n}\right) \leq \rho\left(f h_{n}\right) \leq$ $K \rho(f) \rho\left(h_{n}\right)$ and we obtain that $f\left(t_{n}-1\right) \leq K \rho(f)$, which is a contradiction.

Remark. We note that part (c) of Theorem 1, is not a consequence of [4] or [5], because they used that $A_{\rho}=C(\Omega)$. Also, the example given in the introduction allows us to observe that monotonicity does not implies quasisubmultiplicative. In fact, $\rho$ is not quasi-submultiplicative, otherwise by (c) of Theorem 1, the set $A_{\rho}$ should not admit an unbounded function, which is false. On the other hand there exist non-monotone quasi-submultiplicative norms. In order to see this, let $\rho$ be the Minkowski's functional associated to a bounded balanced convex absorbing set $P$ in $\mathbb{R}^{2}$. If in addition $P$ is a closed set in $\mathbb{R}^{2}$, it is not difficult to see that $\rho$ is monotone if and only if $P$ is symmetric, i.e., if $\left(x_{1}, x_{2}\right) \in P$, then $\left(\epsilon_{1} x_{1}, \epsilon_{2} x_{2}\right) \in P$ where $\epsilon_{i}= \pm 1, i=1,2$. Then if we consider a set $P$ nonsymmetric, the norm $\rho$ is not monotone. However it is quasi-submultiplicative, since $\rho$ is equivalent to the submultiplicative norm $\|\cdot\|_{\infty}$.

In the remainder of this section we study existence and characterization of multiplicative factors.

Theorem 2. Let $\rho$ be a function norm on $C(\Omega)$.If any of the two conditions holds

(a) $\rho$ is a complete norm and $A_{\rho}$ is a subalgebra of $C(\Omega)$, or

(b) $A_{\rho}=C(\Omega)$ where $\Omega$ is a $T_{1}$-space with a dense set of isolated points without accumulation points.

Then there exists a constant $K$ such that $\|f\|_{\infty} \leq K \rho(f)$, for all $f \in C(\Omega)$.

Proof. Suppose that (a) holds and the theorem is false. Then there is a nonnegative function sequence $f_{n}$, with $\left\|f_{n}\right\|_{\infty}=a_{n}, \rho\left(f_{n}\right)=1$ and $\sum_{1}^{\infty} \frac{1}{\left(a_{n}\right)^{\frac{1}{2}}}<$ $\infty$. We can assume without lost of generality that $a_{1} \geq 4$ and $a_{n+1}>a_{n}$ for all $n \in \mathbb{N}$. Let $t_{n}$ a sequence in $\Omega$ be such that $f_{n}\left(t_{n}\right)>\frac{a_{n}}{2}$, and set $J_{n}=\left[f_{n}\left(t_{n}\right)-1, f_{n}\left(t_{n}\right)+1\right]$. For each $n \in \mathbb{N}$, let $g_{n}$ be a non negative function in $C(\mathbb{R})$ with $\operatorname{supp}\left(g_{n}\right) \subset J_{n}, g_{n}\left(f_{n}\left(t_{n}\right)\right)=\frac{a_{n}}{4}=\left\|g_{n}\right\|_{\infty}$. We define a function 
$h_{n} \in C(\Omega)$ by $h_{n}(t)=g_{n}\left(f_{n}(t)\right)$. Given $t \in \Omega$, if $f_{n}(t) \in J_{n}$, then

$$
f_{n}(t) \geq f_{n}\left(t_{n}\right)-1>\frac{a_{n}}{2}-1 \geq \frac{a_{n}}{4}=\left\|g_{n}\right\|_{\infty} \geq g_{n}\left(f_{n}(t)\right)=h_{n}(t),
$$

while $f_{n}(t) \notin J_{n}$, implies that $h_{n}(t)=0$. Therefore $h_{n} \leq f_{n}$ and hence $\rho\left(h_{n}\right) \leq$ $\rho\left(f_{n}\right)=1$.

As $\sum_{n=1}^{\infty} \frac{\rho\left(h_{n}\right)}{\sqrt{a_{n}}} \leq \sum_{1}^{\infty} \frac{1}{\frac{\sqrt{a_{n}}}{2}}<\infty$, the function $s_{k}:=\sum_{n=1}^{k} \frac{h_{n}}{\sqrt{a_{n}}}$ belongs to $A_{\rho}$ and $\left(s_{k}\right)$ is a Cauchy sequence. Since $A_{\rho}$ is a complete space, there exists $s \in A_{\rho}$ such that $\rho\left(s_{k}-s\right) \rightarrow 0$. Moreover, as in part (b) of the proof of theorem 4.8 in [3], we have $s \geq s_{k}$, for every $k$. Then

$$
\|s\|_{\infty} \geq\left\|s_{k}\right\|_{\infty} \geq \frac{\left\|h_{k}\right\|_{\infty}}{\sqrt{a_{k}}} \geq \frac{\sqrt{a_{k}}}{4} \rightarrow \infty
$$

Therefore $s$ is not bounded, contrary to (a) of Theorem 1 .

Now we assume (b) and suppose the theorem is false. Then there exists a sequence of functions $f_{n} \in A_{\rho}, f_{n} \geq 0$ such that $\rho\left(f_{n}\right)=1$ and $\left\|f_{n}\right\|_{\infty} \rightarrow \infty$ for $n \rightarrow \infty$. Thus we can get a sequence of isolated points $t_{n}$ such that $f_{n}\left(t_{n}\right) \rightarrow \infty$ and $t_{n}$ has no accumulation points. If $\delta_{n}$ is the characteristic function of the unitary set $\left\{t_{n}\right\}$, then the function $\delta_{n}$ is continuous. We let $h=\sum_{1}^{\infty} f_{n}\left(t_{n}\right) \delta_{n}$. Since the set $\left\{t_{n}: n \in \mathbb{N}\right\}$ has no accumulation points, it follows that $h \in C(\Omega)$. On the other hand, $\|h\|_{\infty} \geq f_{n}\left(t_{n}\right)$ for all $n \in \mathbb{N}$. Therefore $h \notin B(\Omega)$, contrary to part (b) of Theorem 1.

In particular, the hypothesis (b) of Theorem 2 holds on $\Omega$ when $\Omega=\mathbb{Z}$, the set of integers with the discrete topology.

Corollary 3. Let $\rho$ be a function norm on $C(\Omega)$.

(a) If $\rho$ is complete, then $A_{\rho}$ is a subalgebra of $C(\Omega)$ if and only if $\rho$ is quasi-submultiplicative.

(b) If $\Omega$ is a $T_{1}$ space with a dense set of isolated points without accumulation points, such that $A_{\rho}=C(\Omega)$, then $\rho$ is quasi-submultiplicative.

Proof. We only prove (a) since (b) follows by analogous arguments. Suppose that $A_{\rho}$ is a subalgebra of $C(\Omega)$. Let $f, g \in A_{\rho}$. By Theorem 2 there exists a constant $M$ such that $\|h\|_{\infty} \leq M \rho(h)$ for all $h \in C(\Omega)$. It follows that $\rho(f g) \leq$ $\|f\|_{\infty} \rho(g) \leq M \rho(f) \rho(g)$, i.e. $\rho$ is quasi-submultiplicative. The remaining of the statement is obvious.

The condition that $\rho$ be complete cannot be substituted by the weaker condition of $\sigma$-subadditive, though $A_{\rho}=C(\Omega)$, as the next example shows. 
Example. Let $\Omega=[0,1]$ and define a function norm $\rho$ on $C(\Omega)$ by $\rho(f)=$ $\int_{\Omega}|f| d \mu=\|f\|_{1}$. Clearly, $A_{\rho}=C(\Omega)$ and $\rho$ is $\sigma$-subadditive. We can construct a sequence $\left(f_{n}\right) \in C(\Omega)$ such that $\frac{\left\|f_{n}\right\|_{2}}{\left\|f_{n}\right\|_{1}}$ is arbitrarily large. Thus there is no constant $K$ such that $\rho\left(f^{2}\right) \leq K(\rho(f))^{2}$ for all $f \in C(\Omega)$. Consequently $\rho$ is not quasi-submultiplicative.

Next we will give a characterization of the best multiplicative factor. If $f \in C(\Omega)$ and $K$ is a nonnegative real number, we consider the following subset of $\Omega, A(f, K)=\{t \in \Omega: f(t)>K \rho(f)\}$.

Lemma 4. Let $\rho$ be a quasi-submultiplicative function norm on $C(\Omega)$ and let $f \in C(\Omega)$. If $K$ is a multiplicative factor of $\rho$ and $A(f, K)$ is nonempty, then there exists a function $b \in A_{\rho}, b \neq 0$ such that $\rho(b f)=K \rho(b) \rho(f)$.

Proof. We may assume without lost of generality that $f \geq 0$. Since $A(f, K) \neq$ $\emptyset$, the function $f \in A_{\rho}$ is by Theorem 1 a bounded function. Now set

$$
r=\inf \{f(t): t \in A(f, K)\} .
$$

We have two cases, $r>K \rho(f)$ or $r=K \rho(f)$.

In the first case, we take a nonnegative function $g \in C(\mathbb{R})$ such that $g(x)=0$ for $x \leq K \rho(f)$ and $\|g\|_{\infty}=g(r)=r$. Now, we define $b(t)=g(f(t))$ for $t \in \Omega$. Clearly $b \in C(\Omega)$ and $b \neq 0$. If $t \in A(f, K)$, we have $K \rho(f) \leq(f)(t)$, otherwise we have $b(t)=0$. Thus $(b f)(t) \geq K \rho(f) b(t)$ for all $t \in \Omega$.

In the second case there is $t_{0} \in \Omega$ such that $K \rho(f)<f\left(t_{0}\right) \leq\|f\|_{\infty}$. Here we choose a nonnegative function $g \in C(\mathbb{R})$ with $\operatorname{supp}(g) \subset\left[r,\|f\|_{\infty}\right], g\left(f\left(t_{0}\right)\right) \neq$ 0 and $\|g\|_{\infty}=r$. We define a function $b$ in $C(\Omega)$ by $b(t)=g(f(t))$. Then if $t \in A(f, K)$, we have $K \rho(f)<f(t) \leq\|f\|_{\infty}$, while for $t \notin A(f, K)$ we have $b(t)=0$. Again we get $K \rho(f) b(t) \leq(b f)(t)$ for all $t \in \Omega$.

Thus, in both cases we obtain a function $b \in A_{\rho}, b \neq 0$ such that $(b f)(t) \geq$ $K \rho(f) b(t)$ for all $t \in \Omega$. Finally, since $\rho$ is monotone and $K$ is a multiplicative factor we obtain $K \rho(f) \rho(b) \leq \rho(b f) \leq K \rho(f) \rho(b)$, and this concludes the proof.

Theorem 5. Let $\rho$ a quasi-submultiplicative function norm on $C(\Omega)$ with $A_{\rho} \neq\{0\}$. Then the best multiplicative factor is given by (2).

Proof. Since $A_{\rho} \neq\{0\}$, it is easy to see that there exists a best multiplicative factor and it is given by $M=\sup \{\rho(f g): \rho(f) \leq 1, \rho(g) \leq 1\}$ and $M>0$. Now $\rho(f g) \leq\|f\|_{\infty} \rho(g)$ for all $f, g \in A_{\rho}$ which implies that $M \leq M_{\rho}$. We are going to show that $M \geq M_{\rho}$. Let $f \neq 0$ be a function in $A_{\rho}$ and $\epsilon>0$. Then the set $A(f, M+\epsilon)$ is empty. In fact if this where not so, by Lemma 4 there exists $b \in A_{\rho}, b \neq 0$ such that $\rho(b f)=(M+\epsilon) \rho(b) \rho(f)$, which is contradiction. 
Hence, we must have $A(f, M+\epsilon)=\emptyset$. Therefore $\|f\|_{\infty} \leq(M+\epsilon) \rho(f)$. Thus $M_{\rho} \leq M+\epsilon$, for every $\epsilon>0$.

Corollary 6. Let $\rho$ be a function norm on $C(\Omega)$ with $A_{\rho} \neq\{0\}$ and which satisfies the conditions (a) or (b) of Theorem 2. Then $\rho$ is quasi-submultiplicative and the best multiplicative factor is given by (2).

Proof. It follows immediately from Corollary 3 and Theorem 5.

Corollary 7. Let $\rho$ be a norm on $C(\Omega)$ and $A_{\rho} \neq\{0\}$. Then $\rho$ is quasisubmultiplicative (submultiplicative) if and only if $M_{\rho}<\infty,\left(M_{\rho} \leq 1\right)$. Moreover if $M_{\rho}<\infty$ and $\lambda>0$, the function norm $\lambda \rho$ is submultiplicative if and only if $\lambda \geq M_{\rho}$.

Proof. If $M_{\rho}<\infty,\left(M_{\rho} \leq 1\right)$ the monotonicity of $\rho$ implies that $\rho$ is quasi-submultiplicative (submultiplicative). The converse statement follows by Theorem 5 . Observe that $\lambda>0$ and $A_{\rho} \neq\{0\}$. Then $M_{\lambda \rho}=\frac{1}{\lambda} M_{\rho}$. Thus the proof is completed.

Theorem 8. (a) Let $\rho$ be a function norm on $C(\Omega)$. If $1 \in A_{\rho}$ and $M_{\rho}$ satisfies $\rho(1) M_{\rho}=1$, then $\|f\|_{\infty}=M_{\rho} \rho(f)$, for all $f \in C(\Omega)$.

(b) The infinity norm is the unique submultiplicative function norm on $C(\Omega)$ such that $\rho(1)=1$.

(c) Every quasi-submultiplicative function norm $\rho$ on $C(\Omega)$ such that $1 \in A_{\rho}$, is equivalent to infinity norm.

Proof. Since $\|f\|_{\infty} \leq M_{\rho} \rho(f) \leq M_{\rho} \rho(1)\|f\|_{\infty}$, we have (a).

Now, by Theorem 5 the best multiplicative factor for a quasi-submultiplicative function norm $\rho$ is given by $M_{\rho}$. As $\rho$ is submultiplicative $M_{\rho} \leq 1$. On the other hand, since $\rho(1) \leq M_{\rho}(\rho(1))^{2}$ and $\rho(1)=1$, we have $M_{\rho} \geq 1$. Thus, (b) follows from (a). Finally (c) is a direct consequence of Theorem 5 and of the monotonicity of $\rho$.

\section{References}

[1] R. Arens, M. Goldberg and W. A. Luxemburg, multiplicative factors for function norms, J. Math. Anal. Appl., 177, (1993), 368-385.

[2] H. H. Cuenya, multiplicative factors for function seminorms, J. Math. Anal. Appl., 195 (1995), 440-448.

[3] W. A. J. Luxemburg, A. C. Zaanen, Notes on Banach functions spaces, I, Indag.Math., 25 (1963), 135-147. 
[4] M. J. Meyer, Some algebras without submultiplicative norms or positive functionals, Studia Math., 116(3) (1995), 299-302.

[5] A. R. Pruss, A remark on non existence of an algebra norm for the algebra of continuous functions on a topological space admitting an unbounded continuous function, Studia Math., 116 (3) (1995), 295-297.

[6] B. Yood, On the non existence of norms for some algebras of functions, Studia Math., 111(1) (1994) 97-101. 